\title{
NECROTIZING MYOPATHY PHENOTYPE IN A PATIENT WITH ACTIVE ANTISYNTHETASE SYNDROME
}

\begin{abstract}
João Calvino Soares de Oliveira ${ }^{1, *}$, Guilherme Guimarães Moreira Balbi ${ }^{1}$, André Silva Franco ${ }^{1}$, Renata Lys Pinheiro de Mello1, Victor Caires Tadeu ${ }^{1}$, Henrique Ayres Mayrink Giardini ${ }^{1}$, Lissiane Karine Noronha Guedes ${ }^{1}$, Rosa Maria Rodrigues Pereira ${ }^{1}$, Samuel Katsuyuki Shinjo ${ }^{1}$
\end{abstract}

1.Universidade de São Paulo, São Paulo (SP), Brazil.

*Corresponding author: calvinomed@gmail.com

\section{BACKGROUND}

Antisynthetase syndrome is a systemic autoimmune myopathy characterized by interstitial lung disease, myositis, arthritis, as well as fever, Raynaud's phenomenon, and "mechanic's hands". Laboratory data is characterized by the present of autoantibodies against aminoacyl transfer RNA synthetases. In clinical practice, some clinical features have been known to predominate with different intensities. Herein, we report a patient with antisynthetase syndrome with severe muscle activity that resembled immunemediated necrotizing myopathy.

\section{CASE REPORT}

A 51-year-old white female patient, previously healthy, was referred to our hospital with a two-day history of shoulder and hip pain, as well as proximal muscle weakness, evolving rapidly and aggressively to immobility, associated with elevated of muscle enzymes (creatine phosphokinase 15,168 U/L). Systematic interrogatory demonstrated polyarthralgia, dysphagia, and lower leg vasculitis. Complementary investigation showed a strongly positive anti-Jo-1 antibody, a negative anti-SRP, and a thigh magnetic resonance image with intense edema area. During hospitalization, the patient required orotracheal intubation due to respiratory muscle failure. Treatment with intravenous immunoglobulin $(2 \mathrm{~g} / \mathrm{kg}$ ) and methylprednisolone pulse therapy $(3 \mathrm{~g})$ was performed, evolving to a satisfactory clinical response, and resulting in maintenance therapy with azathioprine and oral prednisone. During the course of treatment, the patient developed autoimmune microangiopathic hemolytic anemia associated with KDIGO III acute kidney injury and subnephrotic proteinuria, which required plasmapheresis and a new intravenous methylprednisolone course. Kidney biopsy was indicated, but was not performed due to complete resolution of the renal condition (improvement of renal function and negative proteinuria), which was attributed to the adverse effect of intravenous immunoglobulin. The patient's clinical and laboratory data improved, and the patient was discharged with oral azathioprine and prednisone tapering.

\section{CONCLUSION}

The clinical presentation of patients with rapidly progressive muscle involvement could be catastrophic, necessitating quick recognition and proper treatment by a rheumatologist. In these cases, the presence of anti-Jo- 1 antibody and quick myopathy progression are factors indicating a predisposition for relapse. In the acute phase, there is a classic response to intravenous methylprednisolone pulse therapy and intravenous immunoglobulin.

\section{KEYWORDS}

Antisynthetase syndrome, Necrotizing myopathy, Myositis. 